\title{
A First Digit Theorem for Square-Free Integer Powers
}

\author{
Werner Hürlimann
}

Feldstrasse 145, CH-8004 Zürich, Switzerland

Copyright (C) 2014 Werner Hürlimann. This is an open access article distributed under the Creative Commons Attribution License, which permits unrestricted use, distribution, and reproduction in any medium, provided the original work is properly cited.

\begin{abstract}
For any fixed integer power, it is shown that the first digits of square-free integer powers follow a generalized Benford law (GBL) with size-dependent exponent that converges asymptotically to a GBL with inverse power exponent. In particular, asymptotically as the power goes to infinity the sequences of squarefree integer powers obey Benford's law. Moreover, we show the existence of a one-parametric size-dependent exponent function that converge to these GBL's and determine an optimal value that minimizes its deviation to two minimum estimators of the size-dependent exponent over the finite range of square-free integer powers less than $10^{s \cdot m}, m=4, \ldots, 10$, where $s=1,2,3,4,5,10$ is a fixed integer power.
\end{abstract}

Mathematics Subject Classification: Primary 11A25, 11K36, 11N37, 11Y55; Secondary 62E20, 62F 12

Keywords: first digit; square-free number; asymptotic counting function; probabilistic number theory; generalized Benford law; mean absolute deviation; probability weighted least squares

\section{Introduction}

It is well-known that the first digits of many numerical data sets are not uniformly distributed. Newcomb [14] and Benford [3] observed that the first digits of many series of real numbers obey Benford's law

$$
P^{B}(d)=\log _{10}(1+d)-\log _{10}(d), \quad d=1,2, \ldots, 9
$$


The increasing knowledge about Benford's law and its applications has been collected in various bibliographies, the most recent being Beebe [2] and Berger and Hill [4]. It is also known that for any fixed power exponent $s \geq 1$, the first digits of integer powers, follow asymptotically a Generalized Benford law (GBL) with exponent $\alpha=s^{-1} \in(0,1)$ such that (see Hürlimann [7])

$$
P_{\alpha}^{G B}(d)=\frac{(1+d)^{\alpha}-d^{\alpha}}{10^{\alpha}-1}, \quad d=1,2, \ldots, 9
$$

Clearly, the limiting case $\alpha \rightarrow 0$ respectively $\alpha \rightarrow 1$ of (1.2) converges weakly to Benford's law respectively the uniform distribution.

We study the distribution of first digits of square-free integer powers. The method consists to fit the GBL to samples of first digits using two size-dependent goodness-of-fit measures, namely the ETA measure (derived from the mean absolute deviation) and the WLS measure (weighted least square measure). In Section 2, we determine the minimum ETA and WLS estimators of the GBL over finite ranges of square-free powers up to $10^{s \cdot m}, m \geq 4, s \geq 1$ a fixed power exponent. Computations illustrate the convergence of the size-dependent GBL with minimum ETA and WLS estimators to the GBL with exponent $s^{-1}$. Moreover, we show the existence of a one-parametric size-dependent exponent function that converge to these GBL's and determine an optimal value that minimizes its deviation to the minimum ETA and WLS estimators. A mathematical proof of the asymptotic convergence of the finite sequences to the GBL with inverse power exponent follows in Section 3.

\section{Size-dependent GBL for square-free integer powers}

To investigate the optimal fitting of the GBL to first digit sequences of squarefree integer powers, it is necessary to specify goodness-of-fit (GoF) measures according to which optimality should hold. First of all, a reasonable GoF measure for the fitting of first-digit distributions should be size-dependent. This has been observed by Furlan [5], Section II.7.1, pp.70-71, who defines the ETA measure, and by Hürlimann [8], p.8, who applies the probability weighted least squares (WLS) measure used earlier by Leemis et al. [12] (chi-square divided by sample size). Let $\left\{x_{n}\right\} \subset[1, \infty), n \geq 1$, be an integer sequence, and let $d_{n}$ be the (first) significant digit of $x_{n}$. The number of $x_{n}$ 's, $n=1, \ldots, N$, with significant digit $d_{n}=d$ is denoted by $X_{N}(d)$. Then, Furlan's ETA measure for the GBL is defined to be 


$$
\operatorname{ETA}_{N}(\alpha)=\frac{9}{2 \cdot N} \cdot M A D_{N}(\alpha), \quad M A D_{N}(\alpha)=\frac{1}{9} \cdot \sum_{d=1}^{9}\left|P_{\alpha}^{G B}(d)-\frac{X_{N}(d)}{N}\right|,
$$

where $M A D_{N}(\alpha)$ is the mean absolute deviation measure. The latter measure is also used to assess conformity to Benford's law by Nigrini [15] (see also Nigrini [16], Table 7.1, p.160). The WLS measure for the GBL is defined by (e.g. [12])

$$
W L S_{N}(\alpha)=\frac{1}{N} \cdot \sum_{d=1}^{9} \frac{\left(P_{\alpha}^{G B}(d)-\frac{X_{N}(d)}{N}\right)^{2}}{P_{\alpha}^{G B}(d)}
$$

Consider now the sequence of square-free integer powers $\left\{n_{f}^{s}\right\}, n_{f}^{s}<10^{s \cdot m}$, for a fixed power exponent $s=1,2,3, \ldots$, and arbitrary square-free numbers $n_{f}$ below $10^{m}, m \geq 4$. Denote by $I_{k}^{s}(d)$ the number of square-free powers below $10^{k}, k \geq 1$, with first digit $d$. This number is defined recursively by the relationship

$$
I_{k+1}^{s}(d)=S\left(\sqrt[s]{(d+1) \cdot 10^{k}}\right)-S\left(\sqrt[s]{d \cdot 10^{k}}\right)+I_{k}^{s}(d), \quad k=1,2, \ldots
$$

where the counting function $S(n)$ is given by (e.g. Pawlewicz [18], Theorem 1)

$$
S(n)=\sum_{k=1}^{\lfloor\sqrt{n}\rfloor} \mu(k) \cdot\left\lfloor\frac{n}{k^{2}}\right\rfloor,
$$

where $\mu(k)$ is the Möbius function such that $\mu(k)=0$ if $p^{2}$ divides $k$ and $\mu(k)=(-1)^{e}$ if $k$ is a square-free number with $e$ distinct prime factors, and $\lfloor\cdot\rfloor$ denotes the integer-part function. Recent algorithms to efficiently compute these arithmetic functions are contained in Pawlewicz [18] and Auil [1].

Therefore, with $N=S\left(10^{m}\right)$ one has $X_{N}(d)=I_{s \cdot m}^{s}(d)$ in (2.1)-(2.2). A list of the $I_{s \cdot m}^{s}(d), m=4, \ldots, 10, s=1,2,3,4,5,10$, together with the sample size $N=S\left(10^{m}\right)$, is provided in Table A.1 of the Appendix. Based on this we have calculated the optimal parameters which minimize the ETA (or equivalently MAD) and WLS measures, the so-called minimum ETA (or minimum MAD) and minimum WLS estimators. Together with their GoF measures, these optimal estimators are reported in Table 2.1 below. Note that the minimum WLS is a critical point of the equation 


$$
\begin{aligned}
& \frac{\partial}{\partial \alpha} W L S_{N}(\alpha)=\frac{1}{N} \cdot \sum_{d=1}^{9} \frac{\partial P_{\alpha}^{G B}(d)}{\partial \alpha} \cdot \frac{P_{\alpha}^{G B}(d)^{2}-\left(\frac{X_{N}(d)}{N}\right)^{2}}{P_{\alpha}^{G B}(d)^{2}}=0 \\
& \frac{\partial P_{\alpha}^{G B}(d)}{\partial \alpha}=\frac{(1+d)^{\alpha}\left\{\ln \left(\frac{1+d}{10}\right) 10^{\alpha}-\ln (1+d)\right\}-d^{\alpha}\left\{\ln \left(\frac{d}{10}\right) 10^{\alpha}-\ln (d)\right\}}{\left(10^{\alpha}-1\right)^{2}} .
\end{aligned}
$$

For comparison, the ETA and WLS measures for the size-dependent GBL exponent

$$
\alpha_{L L}(s \cdot m)=s^{-1} \cdot\left\{1-c \cdot 10^{-m}\right\}
$$

with $c=1$, called LL estimator, are listed. This type of estimator is named in honour of Luque and Lacasa [13] who introduced it in their GBL analysis for the prime number sequence. Through calculation one observes that the LL estimator minimizes the absolute deviations between the LL estimator and the ETA (resp. WLS) estimators over the finite ranges of square-free powers $\left[1,10^{s \cdot m}\right], m=4, \ldots, 10, s=1,2,3,4,5,10$. In fact, if one denotes the ETA and WLS estimators of the sequence $\left\{n_{f}^{s}\right\}, n_{f}^{s}<10^{s \cdot m}$, by $\alpha_{E T A}(s \cdot m)$ and $\alpha_{W L S}(s \cdot m)$, then one has uniformly over the considered finite ranges (consult the columns " $\Delta$ to LL estimate" in Table 2.1 in units of $\left.10^{-(m-3)}\right)$

$$
\begin{aligned}
& \left|\alpha_{W L S}(s \cdot m)-\alpha_{L L}(s \cdot m)\right| \leq 1.96 \cdot 10^{-(m-3)}, \\
& \left|\alpha_{E T A}(s \cdot m)-\alpha_{L L}(s \cdot m)\right| \leq 2.53 \cdot 10^{-(m-3)} .
\end{aligned}
$$

Table 2.1 displays exact results obtained on a computer with single precision, i.e.

\begin{tabular}{|c|c|c|c|c|c|c|c|c|c|c|}
\hline$=1$ & \multicolumn{2}{|c|}{ parameters } & \multicolumn{2}{|c|}{$\Delta$ to LL estimate } & \multicolumn{3}{|c|}{ ETA GoF measures } & \multicolumn{3}{|c|}{ WLS GoF measures } \\
\hline $\mathrm{m}=$ & ILS & |ETA & WLS & |ETA & LL & WLS & ETA & LL & WLS & ETA \\
\hline 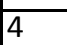 & 9989269 & 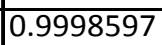 & $\sqrt{10}$ & & 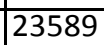 & & & 2263 & 2211 & 250 \\
\hline & 01437 & & 15 & & 13 & 7766 & 761 & 2128 & 2115 & 120 \\
\hline & 393 & & & & & & & 79.5 & 470.5 & 36.2 \\
\hline & 0029 & & & & 5.3 & 275.7 & & 7.5 & 227.1 & 50.0 \\
\hline & 003 & & & & & 32 & & 47 & 36 & \\
\hline & 999 & & 0.069 & & $6 /$ & 4.8 & & 488 & 7.462 & 59 \\
\hline 10 & 0000 & & 0.342 & 0.563 & 418 & 0.339 & $0.5<0$ & 0.665 & 0.600 & 0.627 \\
\hline$s=2$ & \multicolumn{2}{|c|}{ parameters } & \multicolumn{2}{|c|}{$\Delta$ to $L L$ estimate } & \multicolumn{3}{|c|}{ ETA GoF measures } & \multicolumn{3}{|c|}{ WLS GoF measures } \\
\hline $\mathrm{m}=$ & WLS & ETA & WLS & |ETA & LL & WLS & ETA & LL & WLS & ET \\
\hline 4 & 0.4998130 & 0.49 & 0.001 & & 45748 & 45600 & 42296 & 7545 & 7543 & 8777 \\
\hline & & & 0.008 & & 5652 & 5571 & 5240 & 1350 & 346 & 1473 \\
\hline & & & 0.103 & & 875 & 743 & 68 & 272.5 & 201.9 & 216.6 \\
\hline & & & 0.1 & & 220.5 & 192.3 & & 177.8 & 155.7 & 155.7 \\
\hline & & & 0.3 & & 5.13 & 46.47 & & 95.58 & 89.00 & 89.61 \\
\hline 9 & 0.5 & 0.49 & 0.17 & & 7.255 & 7.343 & 7.206 & 22.13 & 21. & 22.42 \\
\hline 10 & 0.5000000 & 0.4999999 & 285 & & 2.033 & 2.054 & 1.990 & 15.5 & 15 & 16.03 \\
\hline
\end{tabular}
with 15 significant digits. The ETA (resp. WLS) measures are given in units of $10^{-(m+7)}$ (resp. $10^{-(2 m+4)}$ ). Taking into account the decreasing units, one observes that the optimal ETA and WLS measures decrease with increasing sample size.

Table 2.1: GBL fit for first digit of square-free powers: ETA vs. WLS criterion 


\begin{tabular}{|c|c|c|c|c|c|c|c|c|c|c|}
\hline \multirow{2}{*}{$\begin{array}{l}\mathrm{s}=3 \\
\mathrm{~m}=\end{array}$} & \multicolumn{2}{|c|}{ parameters } & \multicolumn{2}{|c|}{$\Delta$ to LL estimate } & \multicolumn{3}{|c|}{ ETA GoF measures } & \multicolumn{3}{|c|}{ WLS GoF measures } \\
\hline & WLS & ETA & WLS & ETA & LL & WLS & ETA & LL & WLS & ETA \\
\hline 4 & 0.3321846 & 0.3353504 & 0.011 & 0.021 & 48745 & 50462 & 46805 & 10247 & 10162 & 10847 \\
\hline 5 & 0.3341766 & 0.3347193 & 0.085 & 0.139 & 11481 & 10177 & 9342 & 3766 & 3275 & 3477 \\
\hline 6 & 0.3333554 & 0.3333366 & 0.022 & 0.004 & 1508 & 1508 & 1505 & 955.2 & 951.7 & 954.1 \\
\hline 7 & 0.3333469 & 0.3333720 & 0.136 & 0.387 & 281.4 & 262.7 & 240.4 & 263.2 & 250.6 & 293.8 \\
\hline 8 & 0.3333364 & 0.3333380 & 0.309 & 0.469 & 59.26 & 53.74 & 51.27 & 137.89 & 131.35 & 133.11 \\
\hline 9 & 0.3333338 & 0.3333343 & 0.457 & 0.959 & 8.909 & 8.156 & 7.329 & 27.433 & 26.006 & 27.73 \\
\hline 10 & 0.3333335 & 0.3333336 & 1.951 & 2.308 & 1.957 & 1.677 & 1.625 & 11.456 & 8.850 & 8.937 \\
\hline
\end{tabular}

\begin{tabular}{|l|l|l|l|l|lll|lll|}
\hline $\mathrm{s}=4$ & \multicolumn{3}{|c|}{ parameters } & \multicolumn{2}{|c|}{$\Delta$ to LL estimate } & \multicolumn{3}{c|}{ ETA GoF measures } & \multicolumn{3}{c|}{ WLS GoF measures } \\
$\mathrm{m}=$ & WLS & ETA & WLS & ETA & LL & WLS & ETA & LL & WLS & ETA \\
\hline 4 & 0.2462798 & 0.2505388 & 0.037 & 0.006 & 47287 & 51150 & $\mathbf{4 6 9 6 3}$ & 9747 & $\mathbf{8 8 0 4}$ & 10056 \\
5 & 0.2502442 & 0.2513916 & 0.025 & 0.139 & 9426 & 9198 & $\mathbf{8 5 7 6}$ & $\mathbf{2 4 6 9}$ & $\mathbf{2 4 2 7}$ & 3338 \\
6 & 0.2501231 & 0.2502287 & 0.123 & 0.229 & 1231 & 1100 & $\mathbf{1 0 6 7}$ & 596.0 & $\mathbf{4 9 0 . 6}$ & 567.7 \\
7 & 0.2500166 & 0.2500123 & 0.167 & 0.123 & 242.6 & 208.3 & $\mathbf{2 0 5 . 7}$ & 202.8 & $\mathbf{1 8 3 . 6}$ & 184.9 \\
8 & 0.2500056 & 0.2500018 & 0.560 & 0.179 & 37.28 & 34.64 & $\mathbf{3 1 . 5 9}$ & 79.65 & $\mathbf{5 7 . 9 4}$ & 68.00 \\
9 & 0.2499995 & 0.2499989 & 0.544 & 1.070 & 7.554 & 6.745 & $\mathbf{5 . 9 8 0}$ & 19.206 & $\mathbf{1 7 . 1 5 8}$ & 19.08 \\
10 & 0.2499999 & 0.2500000 & 0.609 & 0.252 & 0.767 & 0.801 & $\mathbf{0 . 7 3 6}$ & $\mathbf{2 . 8 0 9}$ & $\mathbf{2 . 5 5 2}$ & $\mathbf{2 . 6 4 0}$ \\
\hline
\end{tabular}

\begin{tabular}{|l|l|l|l|l|lll|lll|}
\hline $\mathrm{s}=5$ & \multicolumn{3}{|c|}{ parameters } & \multicolumn{2}{|c|}{$\Delta$ to LL estimate } & \multicolumn{3}{c|}{ ETA GoF measures } & \multicolumn{3}{c|}{ WLS GoF measures } \\
$\mathrm{m}=$ & WLS & ETA & WLS & ETA & LL & WLS & ETA & LL & WLS & ETA \\
\hline 4 & 0.2016366 & 0.2040166 & 0.017 & 0.040 & 38498 & 35357 & $\mathbf{3 1 5 2 3}$ & 5066 & $\mathbf{4 8 7 5}$ & 5269 \\
5 & 0.1997147 & 0.1995436 & 0.028 & 0.045 & 4312 & 3471 & $\mathbf{3 0 8 5}$ & 680.7 & $\mathbf{6 2 4 . 9}$ & 645 \\
6 & 0.1999168 & 0.1998905 & 0.083 & 0.109 & 940 & 792 & $\mathbf{7 5 7}$ & 338.9 & $\mathbf{2 9 1 . 0}$ & 295.8 \\
7 & 0.2000150 & 0.2000089 & 0.150 & 0.089 & 196.2 & 179.5 & $\mathbf{1 7 1 . 4}$ & 151.5 & $\mathbf{1 3 5 . 8}$ & 138.4 \\
8 & 0.1999998 & 0.1999991 & 0.022 & 0.093 & 18.69 & 18.42 & $\mathbf{1 7 . 5 5}$ & 14.57 & $\mathbf{1 4 . 5 4}$ & 14.89 \\
9 & 0.1999997 & 0.1999992 & 0.267 & 0.755 & 12.556 & 11.993 & $\mathbf{1 1 . 0 6 7}$ & 49.591 & $\mathbf{4 9 . 0 9 6}$ & 50.75 \\
10 & 0.2000001 & 0.2000001 & 0.501 & 0.639 & 2.091 & 2.014 & $\mathbf{1 . 9 9 3}$ & 21.817 & $\mathbf{2 1 . 6 4 2}$ & 21.655 \\
\hline
\end{tabular}

\begin{tabular}{|l|l|l|l|l|lll|lll|}
\hline $\mathrm{s}=10$ & \multicolumn{3}{|c|}{ parameters } & \multicolumn{2}{|c|}{$\Delta$ to LL estimate } & \multicolumn{3}{c|}{ ETA GoF measures } & \multicolumn{3}{c|}{ WLS GoF measures } \\
$\mathrm{m}=$ & WLS & ETA & WLS & ETA & LL & WLS & ETA & LL & WLS & ETA \\
\hline 4 & 0.1084757 & 0.1078678 & 0.085 & 0.079 & 44303 & 33110 & $\mathbf{3 1 3 6 7}$ & 12124 & $\mathbf{7 0 8 5}$ & $\mathbf{7 1 1 1}$ \\
5 & 0.0999163 & 0.0999959 & 0.008 & 0.000 & 5865 & 6040 & $\mathbf{5 8 6 0}$ & 1705 & $\mathbf{1 7 0 0}$ & 1705 \\
6 & 0.0999481 & 0.0999054 & 0.052 & 0.094 & 1551 & 1487 & $\mathbf{1 4 3 4}$ & 818.3 & $\mathbf{7 9 9 . 5}$ & 812.2 \\
7 & 0.1000192 & 0.1000119 & 0.192 & 0.119 & 248.8 & 220.4 & $\mathbf{2 1 2 . 9}$ & 289.0 & $\mathbf{2 6 3 . 2}$ & 266.9 \\
8 & 0.1000013 & 0.1000002 & 0.134 & 0.024 & 46.86 & 48.36 & $\mathbf{4 6 . 3 7}$ & 120.76 & $\mathbf{1 1 9 . 5 0}$ & 120.34 \\
9 & 0.0999994 & 0.0999997 & 0.624 & 0.289 & 10.394 & 9.661 & $\mathbf{9 . 4 7 9}$ & 36.219 & $\mathbf{3 3 . 4 9 7}$ & 34.28 \\
10 & 0.1000002 & 0.1000003 & 1.541 & 2.526 & 2.772 & 2.474 & $\mathbf{2 . 3 6 1}$ & 23.292 & $\mathbf{2 1 . 6 3 1}$ & 22.310 \\
\hline
\end{tabular}

\section{Asymptotic counting function for square-free integer powers}

The following is a slight extension of the argument by Luque and Lacasa [13], Section 5(a). It is well-known that a random process with uniform density $x^{-1}$ generates data that are Benford distributed. Similarly, a sequence of numbers 
generated by a power-law density $x^{-\alpha}, \alpha \in(0,1)$, has a GBL first-digit distribution $P_{1-\alpha}^{G B}(d)$ with exponent $1-\alpha$. From such a density it is possible to derive a counting function $C(N)$ for that sequence in the interval $[1, N]$. However, assuming a local density of the form $x^{-\alpha(x)}$ such that $C(N) \sim \int_{2}^{N} x^{-\alpha(x)} d x$ is not appropriate in general. Indeed, the square-free power relation over an interval $\left[1, N^{s}\right]$ that belongs to (2.6), namely

$$
\alpha\left(N^{s}\right)=\frac{s-1+\alpha(N)}{s}, \quad \alpha(N)=\frac{C}{N},
$$

does not behave smoothly in $\left[1, N^{s}\right]$, which should be the case for such an approximation. This drawback can be overcome. Denote by $Q_{s}\left(N^{s}\right)$ the counting function for square-free powers in $\left[1, N^{s}\right]$. Instead of $\int_{2}^{N^{s}} x^{-\alpha\left(N^{s}\right)} d x$ define

$$
Q_{s}\left(N^{s}\right)=\frac{6}{\pi^{2} \cdot s} \cdot \int_{2}^{N^{s}} x^{-\alpha\left(N^{s}\right)} d x
$$

where the integral pre-factor is chosen to fulfill the asymptotic limiting value for the square-free number counting function, that is (note that $n_{f}^{s}<N^{s}$ if, and only if, one has $n_{f}<N$ )

$$
\lim _{N \rightarrow \infty} \frac{Q_{s}\left(N^{s}\right)}{N}=\frac{6}{\pi^{2}}
$$

In fact, two improved asymptotic expansions of $S(N)$ are known, namely

$$
S(N)=\frac{6}{\pi^{2}} N+O(\sqrt{N}), \text { and } S(N)=\frac{6}{\pi^{2}} N+O\left(N^{\frac{17}{54}+\varepsilon}\right)
$$

The first one is classical and proved in Hardy and Wright [6], p.269, and Jameson [9], Section 2.5, for example. The second improved estimate is due to Jia [11] (see also Pappalardi [17]). However, it suffices to use the simple estimate (3.3), which is obtained as follows. From (3.2) one gets for arbitrary $s=1,2, \ldots$

$$
Q_{s}\left(N^{s}\right)=\frac{6}{\pi^{2} \cdot s} \cdot \int_{2}^{N^{s}} x^{-\alpha\left(N^{s}\right)} d x=\frac{6}{\pi^{2}} \cdot \frac{1}{s \cdot\left(1-\alpha\left(N^{s}\right)\right)} \cdot N^{s \cdot\left(1-\alpha\left(N^{s}\right)\right)} .
$$

With (3.1) this transforms to 


$$
Q_{s}\left(N^{s}\right)=\frac{6}{\pi^{2}} \cdot \frac{1}{1-\alpha(N)} \cdot N^{1-\alpha(N)}=\frac{6}{\pi^{2}} \cdot N \cdot \frac{N}{N-c} \cdot \exp \left(-c \frac{\ln (N)}{N}\right),
$$

which is independent of $s$ and simply denoted by $Q(N)$. The equality $Q_{s}\left(N^{s}\right)=Q(N) \quad$ reflects the fact that there are as many square-free powers in $\left[1, N^{s}\right]$ as there are square-free numbers in $[1, N]$. Now, what is a good value of $c \in[1, N)$ ? Clearly, the factor

$$
f_{N}(c)=\frac{N}{N-c} \cdot \exp \left(-c \frac{\ln (N)}{N}\right)
$$

converges to 1 as $N \rightarrow \infty$ for any fixed $C$. Its derivative with respect to $C$ satisfies the property

$$
\frac{\partial}{\partial c} f_{N}(c)<0, \quad \forall c \in\left[1, \frac{\ln (N)-1}{\ln (N)} N\right) \subseteq[1, N), \quad \forall N \geq 4,
$$

which implies the following min-max property of (3.7) at $c=1$ :

$$
\min _{N \geq 10^{4}}\left\{\max _{c \in\left[1, \frac{\ln (N)-1}{\ln (N)} N\right)} f_{N}(c)\right\}=f_{10^{4}}(1)=0.99918 .
$$

The size-dependent exponent (3.1) with $c=1$ not only minimizes the absolute deviations between the LL estimator and the ETA (resp. WLS) estimators over the finite ranges of square-free powers $\left[1,10^{s \cdot m}\right], m=4, \ldots, 10, s=1,2,3,4,5,10$, as shown in Section 2, but it turns out to be uniformly best with maximum error less than $10^{-3}$ against the asymptotic estimate, at least if $N \geq 10^{4}$. Moreover, the following limiting asymptotic result has been obtained.

First Digit Square-Free Integer Power Theorem (GBL for square-free integer powers). The asymptotic distribution of the first digit of square-free integer power sequences $n_{f}^{s}<10^{s \cdot m}, m \geq 4$, for fixed $s=1,2,3, \ldots$, as $m \rightarrow \infty$, is given by

$$
\lim _{m \rightarrow \infty} \frac{I_{s \cdot m}^{s}(d)}{S\left(10^{m}\right)}=\lim _{m \rightarrow \infty} P_{\alpha(s \cdot m)}^{G B}(d)=P_{s^{-1}}^{G B}(d), \quad d=1, \ldots, 9, \quad \alpha(s \cdot m)=\frac{1}{s}\left(1-\frac{1}{10^{m}}\right)
$$

Table 3.1 compares the new counting function $Q(N)=Q_{s}\left(N^{s}\right), \forall s=1,2, \ldots$, with the exact and asymptotic counting functions $S(N)$ and $S_{a s}(N)=\frac{6}{\pi^{2}} N$. 
Table 3.1: Comparison of square-free number counting functions for $N=10^{m}$

\begin{tabular}{|c|c|c|c|c|}
\hline $\mathrm{m}$ & $\mathrm{S}(\mathrm{N})$ & $Q(N)$ & $6 N / \pi^{2}$ & $\mathrm{Q}(\mathrm{N}) / \mathrm{S}(\mathrm{N})$ \\
\hline 1 & 7 & 5 & 6 & 0.7142857142857 \\
\hline 2 & 61 & 58 & 60 & 0.9508196721311 \\
\hline 3 & 608 & 604 & 607 & 0.9934210526316 \\
\hline 4 & 6'083 & 6'074 & 6'079 & 0.9985204668749 \\
\hline 5 & 60'794 & 60'786 & 60'792 & 0.9998684080666 \\
\hline 6 & $607 ' 926$ & 607'919 & $607 ' 927$ & 0.9999884854407 \\
\hline 7 & 6'079'291 & 6'079'261 & 6'079'271 & 0.9999950652140 \\
\hline 8 & 60'792'694 & 60'792'699 & 60'792'710 & 1.0000000822467 \\
\hline 9 & $607 ' 927 ' 124$ & $607^{\prime} 927^{\prime} 089$ & 607'927'101 & 0.9999999424273 \\
\hline 10 & 6'079'270'942 & 6'079'271'005 & 6'079'271'018 & 1.0000000103631 \\
\hline 11 & $60^{\prime} 792^{\prime} 710^{\prime} 280$ & $60 ' 792^{\prime} 710^{\prime} 170$ & 60'792'710'185 & 0.99999999881906 \\
\hline 12 & 607'927'102'274 & 607'927'101'837 & $607 ' 927^{\prime} 101 ' 854$ & 0.99999999992812 \\
\hline 13 & 6'079'271'018'294 & 6'079'271'018'522 & 6'079'271'018'540 & 1.0000000000375 \\
\hline 14 & $60 ' 792$ '710'185'947 & $60 ' 792^{\prime} 710^{\prime} 185^{\prime} 383$ & $60 ' 792 ' 710 ' 185^{\prime} 402$ & 0.99999999999907 \\
\hline 15 & 607'927'101'854'103 & 607'927'101'854'006 & $607^{\prime} 927^{\prime} 101^{\prime} 854^{\prime} 026$ & 0.9999999999998 \\
\hline
\end{tabular}

Concluding Remark 3.1. As proved by Jameson [10] the proportion of odd square-free numbers is asymptotically equal to $4 / \pi^{2}$, from which it follows that the ratio of odd to even square-free numbers is $2: 1$. The interested reader might investigate the corresponding sequences of odd and even square-free integer powers and derive similar GBL results.

Appendix: Tables of first digits for square-free integer powers

Based on the recursive relation (2.3)-(2.4), the computation of $I_{s \cdot m}^{s}(d), m=4, \ldots, 10$, is straightforward, at least if a table of the Möbius function is available (e.g. sequence A008683 in OEIS founded by Sloane [19]). These numbers are listed in Table A.1. The entry $s \rightarrow \infty$ corresponds to the limiting Benford law as the power goes to infinity. 
Table A.1: First digit distribution of square-free powers up to $10^{s \cdot m}, m=4, \ldots, 10$

\begin{tabular}{|l|l|l|l|l|l|l|l|}
\hline $\mathrm{s}=1$ / first digit & $6^{\prime} 083$ & $60^{\prime} 794$ & $607^{\prime} 926$ & $6^{\prime} 079^{\prime} 291$ & $60^{\prime} 792^{\prime} 694$ & $607^{\prime} 927^{\prime} 124$ & $6^{\prime} 079^{\prime} 270^{\prime} 942$ \\
\hline 1 & 676 & $6^{\prime} 753$ & $67^{\prime} 540$ & $675^{\prime} 491$ & $6^{\prime} 754^{\prime} 775$ & $67^{\prime} 547^{\prime} 507$ & $675^{\prime} 474^{\prime} 599$ \\
2 & 677 & $6^{\prime} 759$ & $67^{\prime} 556$ & $675^{\prime} 452$ & $6^{\prime} 754^{\prime} 706$ & $67^{\prime} 547^{\prime} 406$ & $675^{\prime} 474^{\prime} 562$ \\
3 & 677 & $6^{\prime} 745$ & $67^{\prime} 532$ & $675^{\prime} 495$ & $6^{\prime} 754^{\prime} 719$ & $67^{\prime} 547^{\prime} 498$ & $675^{\prime} 474^{\prime} 519$ \\
4 & 677 & $6^{\prime} 768$ & $67^{\prime} 561$ & $675^{\prime} 458$ & $6^{\prime} 754^{\prime} 749$ & $67^{\prime} 547^{\prime} 370$ & $675^{\prime} 474^{\prime} 546$ \\
5 & 671 & $6^{\prime} 743$ & $67^{\prime} 539$ & $675^{\prime} 463$ & $6^{\prime} 754^{\prime} 764$ & $67^{\prime} 547^{\prime} 480$ & $675^{\prime} 474^{\prime} 534$ \\
6 & 679 & $6^{\prime} 762$ & $67^{\prime} 566$ & $675^{\prime} 513$ & $6^{\prime} 754^{\prime} 770$ & $67^{\prime} 547^{\prime} 491$ & $675^{\prime} 474^{\prime} 499$ \\
7 & 678 & $6^{\prime} 749$ & $67^{\prime} 533$ & $675^{\prime} 432$ & $6^{\prime} 754^{\prime} 684$ & $67^{\prime} 547^{\prime} 455$ & $675^{\prime} 474^{\prime} 641$ \\
8 & 672 & $6^{\prime} 758$ & $67^{\prime} 547$ & $675^{\prime} 486$ & $6^{\prime} 754^{\prime} 746$ & $67^{\prime} 547^{\prime} 422$ & $675^{\prime} 474^{\prime} 533$ \\
9 & 676 & $6^{\prime} 757$ & $67^{\prime} 552$ & $675^{\prime} 501$ & $6^{\prime} 754^{\prime} 781$ & $67^{\prime} 547^{\prime} 495$ & $675^{\prime} 474^{\prime} 509$ \\
\hline
\end{tabular}

\begin{tabular}{|l|l|l|l|l|l|l|l|}
\hline $\mathrm{s}=2$ / first digit & $6^{\prime} 083$ & $60^{\prime} 794$ & $607^{\prime} 926$ & $6^{\prime} 079^{\prime} 291$ & $60^{\prime} 792^{\prime} 694$ & $607^{\prime} 927^{\prime} 124$ & $6^{\prime} 079^{\prime} 270^{\prime} 942$ \\
\hline 1 & $1^{\prime} 171$ & $11^{\prime} 652$ & $116^{\prime} 439$ & $1^{\prime} 164^{\prime} 549$ & $11^{\prime} 645^{\prime} 617$ & $116^{\prime} 456^{\prime} 692$ & $1^{\prime} 164^{\prime} 566^{\prime} 728$ \\
2 & 884 & $8^{\prime} 934$ & $89^{\prime} 367$ & $893^{\prime} 612$ & $8^{\prime} 936^{\prime} 003$ & $89^{\prime} 360^{\prime} 203$ & $893^{\prime} 603^{\prime} 026$ \\
3 & 753 & $7^{\prime} 526$ & $75^{\prime} 336$ & $753^{\prime} 361$ & $7^{\prime} 533^{\prime} 536$ & $75^{\prime} 334^{\prime} 407$ & $753^{\prime} 342^{\prime} 957$ \\
4 & 671 & $6^{\prime} 640$ & $66^{\prime} 369$ & $663^{\prime} 684$ & $6^{\prime} 637^{\prime} 031$ & $66^{\prime} 370^{\prime} 825$ & $663^{\prime} 708^{\prime} 054$ \\
5 & 600 & $5^{\prime} 997$ & $59^{\prime} 991$ & $600^{\prime} 024$ & $6^{\prime} 000^{\prime} 331$ & $60^{\prime} 003^{\prime} 694$ & $600^{\prime} 037^{\prime} 812$ \\
6 & 553 & $5^{\prime} 517$ & $55^{\prime} 184$ & $551^{\prime} 776$ & $5^{\prime} 517^{\prime} 885$ & $55^{\prime} 179^{\prime} 201$ & $551^{\prime} 791^{\prime} 957$ \\
7 & 510 & $5^{\prime} 137$ & $51^{\prime} 367$ & $513^{\prime} 610$ & $5^{\prime} 135^{\prime} 979$ & $51^{\prime} 359^{\prime} 553$ & $513^{\prime} 5955^{\prime} 378$ \\
8 & 481 & $4^{\prime} 835$ & $48^{\prime} 249$ & $482^{\prime} 421$ & $4^{\prime} 823^{\prime} 838$ & $48^{\prime} 237^{\prime} 994$ & $482^{\prime} 379^{\prime} 468$ \\
9 & 460 & $4^{\prime} 556$ & $45^{\prime} 624$ & $456^{\prime} 254$ & $4^{\prime} 562^{\prime} 474$ & $45^{\prime} 624^{\prime} 555$ & $456^{\prime} 245 ' 562$ \\
\hline
\end{tabular}

\begin{tabular}{|l|l|l|l|l|l|l|l|}
\hline s=3 / first digit & $6^{\prime} 083$ & $60^{\prime} 794$ & $607^{\prime} 926$ & $6^{\prime} 079^{\prime} 291$ & $60^{\prime} 792^{\prime} 694$ & $607^{\prime} 927^{\prime} 124$ & $6^{\prime} 079^{\prime} 270^{\prime} 942$ \\
\hline 1 & $1^{\prime} 367$ & $13^{\prime} 670$ & $136^{\prime} 857$ & $1^{\prime} 368^{\prime} 703$ & $13^{\prime} 687^{\prime} 419$ & $136^{\prime} 874^{\prime} 710$ & $1^{\prime} 368^{\prime} 747^{\prime} 899$ \\
2 & 971 & $9^{\prime} 615$ & $96^{\prime} 052$ & $960^{\prime} 208$ & $9^{\prime} 601^{\prime} 504$ & $96^{\prime} 014^{\prime} 609$ & $960^{\prime} 145^{\prime} 043$ \\
3 & 765 & $7^{\prime} 647$ & $76^{\prime} 437$ & $764^{\prime} 375$ & $7^{\prime} 643^{\prime} 723$ & $76^{\prime} 4366^{\prime} 951$ & $764^{\prime} 369^{\prime} 702$ \\
4 & 642 & $6^{\prime} 445$ & $64^{\prime} 525$ & $645^{\prime} 443$ & $6^{\prime} 454^{\prime} 686$ & $64^{\prime} 548^{\prime} 120$ & $645^{\prime} 481^{\prime} 279$ \\
5 & 559 & $5^{\prime} 632$ & $56^{\prime} 413$ & $564^{\prime} 238$ & $5^{\prime} 642^{\prime} 255$ & $56^{\prime} 422^{\prime} 496$ & $564^{\prime} 225^{\prime} 563$ \\
6 & 506 & $5^{\prime} 055$ & $50^{\prime} 454$ & $504^{\prime} 532$ & $5^{\prime} 045^{\prime} 423$ & $50^{\prime} 454^{\prime} 044$ & $504^{\prime} 5400^{\prime} 015$ \\
7 & 459 & $4^{\prime} 590$ & $45^{\prime} 848$ & $458^{\prime} 528$ & $4^{\prime} 585^{\prime} 135$ & $45^{\prime} 850^{\prime} 705$ & $458^{\prime} 505^{\prime} 935$ \\
8 & 416 & $4^{\prime} 213$ & $42^{\prime} 169$ & $421^{\prime} 720$ & $4^{\prime} 217^{\prime} 192$ & $42^{\prime} 172^{\prime} 165$ & $421^{\prime} 722^{\prime} 747$ \\
9 & 398 & $3^{\prime} 927$ & $39^{\prime} 171$ & $391^{\prime} 544$ & $3^{\prime} 915^{\prime} 357$ & $39^{\prime} 153^{\prime} 324$ & $391^{\prime} 532^{\prime} 759$ \\
\hline
\end{tabular}

\begin{tabular}{|l|l|l|l|l|l|l|l|}
\hline s=4 / first digit & $6^{\prime} 083$ & $60^{\prime} 794$ & $607^{\prime} 926$ & $6^{\prime} 079^{\prime} 291$ & $60^{\prime} 792^{\prime} 694$ & $607^{\prime} 927^{\prime} 124$ & $6^{\prime} 079^{\prime} 270^{\prime} 942$ \\
\hline 1 & $1^{\prime} 477$ & $14^{\prime} 761$ & $147^{\prime} 762$ & $1^{\prime} 477^{\prime} 917$ & $14^{\prime} 779^{\prime} 258$ & $147^{\prime} 793^{\prime} 000$ & $1^{\prime} 477^{\prime} 928^{\prime} 516$ \\
2 & 999 & $9^{\prime} 920$ & $99^{\prime} 105$ & $990^{\prime} 957$ & $9^{\prime} 909^{\prime} 733$ & $99^{\prime} 097^{\prime} 825$ & $990^{\prime} 978^{\prime} 621$ \\
3 & 776 & $7^{\prime} 677$ & $76^{\prime} 658$ & $766^{\prime} 577$ & $7^{\prime} 665^{\prime} 808$ & $76^{\prime} 658^{\prime} 390$ & $766^{\prime} 584^{\prime} 635$ \\
4 & 630 & $6^{\prime} 344$ & $63^{\prime} 402$ & $633^{\prime} 807$ & $6^{\prime} 337^{\prime} 591$ & $63^{\prime} 376^{\prime} 082$ & $633^{\prime} 760^{\prime} 811$ \\
5 & 546 & $5^{\prime} 449$ & $54^{\prime} 461$ & $544^{\prime} 694$ & $5^{\prime} 447^{\prime} 186$ & $54^{\prime} 471^{\prime} 736$ & $544^{\prime} 717^{\prime} 879$ \\
6 & 472 & $4^{\prime} 797$ & $48^{\prime} 035$ & $480^{\prime} 351$ & $4^{\prime} 803^{\prime} 264$ & $48^{\prime} 032^{\prime} 531$ & $480^{\prime} 3244^{\prime} 229$ \\
7 & 431 & $4^{\prime} 319$ & $43^{\prime} 139$ & $431^{\prime} 315$ & $4{ }^{\prime} 313^{\prime} 052$ & $43^{\prime} 130^{\prime} 290$ & $431^{\prime} 303^{\prime} 473$ \\
8 & 393 & $3^{\prime} 924$ & $39^{\prime} 258$ & $392^{\prime} 585$ & $3^{\prime} 925^{\prime} 720$ & $39^{\prime} 257^{\prime} 307$ & $392^{\prime} 573^{\prime} 396$ \\
9 & 359 & $3^{\prime} 603$ & $36^{\prime} 106$ & $361^{\prime} 088$ & $3^{\prime} 611^{\prime} 082$ & $36^{\prime} 109^{\prime} 963$ & $361^{\prime} 099^{\prime} 382$ \\
\hline
\end{tabular}




\begin{tabular}{|c|c|c|c|c|c|c|c|}
\hline$S=5 /$ first digit & $6^{\prime} 083$ & $60^{\prime} 794$ & $607^{\prime} 926$ & 6'079'291 & 60'792'694 & $607^{\prime} 927^{\prime} 124$ & \begin{tabular}{|l|} 
6'079'270'942 \\
\end{tabular} \\
\hline 1 & 1'541 & $15^{\prime} 462$ & 154 '569 & 1'545'536 & $15^{\prime} 455^{\prime} 439$ & $154^{\prime} 554^{\prime} 413$ & \begin{tabular}{|l|}
$1 ' 545^{\prime} 542 ' 924$ \\
\end{tabular} \\
\hline 2 & 1'016 & 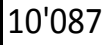 & $100 ' 847$ & 1'008'552 & 10'085'379 & $100 ' 853$ '684 & 1'008'538'705 \\
\hline 3 & 769 & 664 & $76 ' 685$ & $766 ' 804$ & 7'668'233 & 6'682'538 & '201 \\
\hline 4 & 619 & 54 & 162581 & $625^{\prime}$ & 11 & 59 & 3'405 \\
\hline$\tau$ & 3 & 25 & 11 & 33 & 60 & 92 & 123 \\
\hline 6 & 8 & 64 & $46 ' 567$ & $65 ' 699$ & 29 & 56 & 982 \\
\hline 7 & 414 & 153 & $41 ' 502$ & 415'167 & 4'15 & 413 & 4'378 \\
\hline 8 & 378 & 752 & 37'555 & $375 ' 533$ & 3'755'169 & 865 & 375'518'563 \\
\hline 9 & 345 & 33 & 34 '343 & $43^{\prime} 499$ & 13 & 04 & 661 \\
\hline$s=\infty$ & $6^{\prime} 083$ & '794 & $607^{\prime} 926$ & 6'079'291 & 60'792'694 & 7'124 & 6'079 \\
\hline 1 & 1'831 & $18^{\prime} 301$ & $183^{\prime} 004$ & 1'830'049 & $18^{\prime} 300^{\prime} 424$ & $183^{\prime} 004^{\prime} 300$ & $1^{\prime} 830^{\prime} 042^{\prime} 905$ \\
\hline 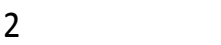 & 1'071 & D'705 & $107^{\prime} 050$ & 1'070'510 & $10^{\prime} 705^{\prime} 062$ & $107^{\prime} 050^{\prime} 653$ & 1'070'506'474 \\
\hline 3 & 760 & 7'596 & 75'954 & 759'539 & 7'595'362 & 75'953'647 & 759'536'431 \\
\hline 4 & 590 & 5'892 & 58'914 & $589 ' 144$ & 5'891'421 & 58'914'225 & $589 ' 142$ '226 \\
\hline 5 & 482 & 4'814 & $48^{\prime} 136$ & 481'366 & 4'813'641 & $48^{\prime} 136^{\prime} 427$ & $481^{\prime} 364^{\prime} 248$ \\
\hline 6 & 407 & 070 & $40 ' 699$ & 406'989 & 4'069'876 & $40 ' 698^{\prime} 769$ & $406 ' 987^{\prime} 673$ \\
\hline 7 & 353 & 26 & $35 ' 255$ & $352 ' 550$ & 3'525'487 & 4'878 & $52 ' 548^{\prime} 758$ \\
\hline 8 & 311 & 3'110 & 31'097 & 310'971 & 3'109'700 & $31^{\prime} 097^{\prime} 006$ & 310'970'043 \\
\hline 0 & 278 & $2 ' 782$ & $27^{\prime} 817$ & $278 ' 173$ & 2'781'721 & $27^{\prime} 817^{\prime} 220$ & $278 ' 172$ '183 \\
\hline
\end{tabular}

\section{References}

[1] F. Auil, An algorithm to generate square-free numbers and to compute the Möbius function, Journal of Number Theory 133, (2013), 426-436.

[2] N.H.F. Beebe, A bibliography of publications about Benford's law, Heap's law, and Zipf's law, (2014), version 1.60 at URL:

ftp://ftp.math.utah.edu/pub/tex/bib/benfords-law.pdf.

[3] F. Benford, The law of anomalous numbers, Proc. Amer. Phil. Soc. 78, (1938), 551-572.

[4] A. Berger and T. Hill, Benford Online Bibliography, 2009.

URL: http://www.benfordonline.net/

[5] L.V. Furlan, Das Harmoniegesetz der Statistik: Eine Untersuchung über die metrische Interdependenz der sozialen Erscheinungen, Basel, Verlag für Recht und Gesellschaft AG, 1946. Reviewed by H. Geiringer, J. Amer. Statist. Assoc. 43(242), (1948), 325-328.

[6] G.H. Hardy and E.M. Wright, An introduction to the theory of numbers, ( $5^{\text {th }}$ ed), Oxford University Press, 1979. 
[7] W. Hürlimann, Integer powers and Benford's law, Int. J. Pure Appl. Math. 11(1), (2004), 39-46.

[8] W. Hürlimann, Generalizing Benford's law using power laws: applications to integer sequences, Int. J. Math. and Math. Sci., (2009), Article ID 970284.

[9] G.J.O. Jameson, The prime number theorem, Cambridge University Press, Cambridge, 2003.

[10] G.J.O. Jameson, Even and odd square-free numbers, Math. Gazette 94, (2010), 123-127.

[11] C.H. Jia, The distribution of square-free numbers, Sci. China Ser. A 36(2), (1993), 154-169.

[12] L.M. Leemis, B.W. Schmeiser and D.L. Evans, Survival distributions satisfying Benford's law, The Amer. Statistician 54(3), (2000), 1-6.

[13] B. Luque and L. Lacasa, The first-digit frequencies of prime numbers and Riemann zeta zeros, Proc. Royal Soc. A 465, (2009), 2197-2216.

[14] S. Newcomb, Note on the frequency of use of the different digits in natural numbers, Amer. J. Math. 4, (1881), 39-40.

[15], M.J. Nigrini, Digital analysis using Benford's law: test statistics for Auditors, Vancouver, Canada, Global Audit Publications, 2000.

[16] M.J. Nigrini, Benford's Law, Applications for forensic accounting, auditing, and fraud detection, J. Wiley \& Sons, Hoboken, New Jersey, 2012.

[17] F. Pappalardi, A survey on k-freeness, In: S.D. Adhikari, R. Balasubramaniam and K. Srinivas (Eds.), Ramanujan Mathematical Society Lecture Notes Series 1, (2004), 71-88.

[18] J. Pawlewicz, Counting square-free numbers, Preprint, (2011), URL: arXiv:1107.4890v1 [math.NT].

[19] N.J.A. Sloane, The On-Line Encyclopedia of Integer Sequences, (1964), URL: https://oeis.org/

\section{Received: June 1, 2014}

